\title{
Erratum to: Effectiveness of the Early Start Denver Model: a Systematic Review
}

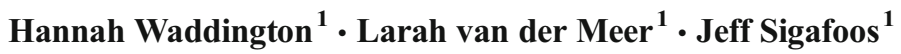

Published online: 27 October 2016

(C) Springer Science+Business Media New York 2016

Erratum to: Review Journal of Autism and Developmental

Disorders June 2016, Volume 3, Issue 2, pp 93-106

DOI 10.1007/s40489-015-0068-3

In the article listed above, the first four child outcomes listed in Table 2 on page 98 were not fully presented. Specifically:

1. The child outcomes reported in Table 2 for the study by Vismara et al. (2008) read "Child's diagnosis changed from ASD to PDD-NOS". However, the complete summary should be: "Spontaneous functional verbal utterances, imitative behaviors, and attentiveness and social initiations (CBRS) increased during intervention and were maintained during follow-up. Child's diagnosis changed from ASD to PDD-NOS".

2. The child outcomes reported in Table 2 for the study by Vismara et al. (2009a) read "Imitative behaviors increased for 7 of 8 children during intervention and this level was maintained for the children who participated in follow-up". However, the complete summary should be: "Spontaneous functional verbal utterances, attentiveness, and social initiations (CBRS) increased for all children during intervention. This was maintained for the 6 children who participated in follow-up. Imitative behaviors increased for 7 of 8 children during intervention and this level was maintained for the children who participated in follow-up".

The online version of the original article can be found at http:// dx.doi.org/10.1007/s40489-015-0068-3.

Hannah Waddington

hannah.waddington@vuw.ac.nz

1 School of Education, Victoria University of Wellington, Karori Campus, PO Box 17-310, Wellington, New Zealand
3. The child outcomes reported in Table 2 for the study by Vismara et al. (2009b) read "There was no significant increase in imitative behaviors". However, the complete summary should be: "Spontaneous functional verbal utterances, attentiveness and social initiations (CBRS) were significantly higher during the didactic training and team supervision conditions. There was no significant increase in imitative behaviors".

4. The child outcomes reported in Table 2 for the study by Dawson et al. (2010) read "The ESDM group were significantly more likely to have improved diagnosis (ASD $\rightarrow$ PDD-NOS)". However, the complete summary should be: "After 2 years, children in the ESDM intervention had significantly higher overall cognitive skills (MSEL), including receptive language and expressive language than the TAU group. They also had significantly higher adaptive behavior (VABS), including communication, daily living skills and motor skills. There were no significant group differences in the visual reception (MSEL), fine motor skills (MSEL), socialization (VABS), autism severity (ADOS), or repetitive behaviors (RBS). The ESDM group were significantly more likely to have improved diagnosis, moving from a diagnosis of ASD to PDD-NOS". 